\title{
RESEARCH ON REMOTE SENSING IMAGE CLASSIFICATION BASED ON FEATURE LEVEL FUSION
}

\author{
Lin Yuan ${ }^{1}$, Guobin Zhu ${ }^{1, *}$ \\ ${ }^{1}$ School of Remote Sensing and Information Engineering, Wuhan University, 430079, Wuhan, China - (yuanlin_0@foxmail.com, \\ gbzhu@whu.edu.cn)
}

KEY WORDS: Remote sensing image fusion, Remote sensing image classification, Feature level, Sentinel-1A, Landsat8 OLI

\begin{abstract}
:
Remote sensing image classification, as an important direction of remote sensing image processing and application, has been widely studied. However, in the process of existing classification algorithms, there still exists the phenomenon of misclassification and missing points, which leads to the final classification accuracy is not high. In this paper, we selected Sentinel-1A and Landsat8 OLI images as data sources, and propose a classification method based on feature level fusion. Compare three kind of feature level fusion algorithms (i.e., Gram-Schmidt spectral sharpening, Principal Component Analysis transform and Brovey transform), and then select the best fused image for the classification experimental. In the classification process, we choose four kinds of image classification algorithms (i.e. Minimum distance, Mahalanobis distance, Support Vector Machine and ISODATA) to do contrast experiment. We use overall classification precision and Kappa coefficient as the classification accuracy evaluation criteria, and the four classification results of fused image are analysed. The experimental results show that the fusion effect of Gram-Schmidt spectral sharpening is better than other methods. In four kinds of classification algorithms, the fused image has the best applicability to Support Vector Machine classification, the overall classification precision is $94.01 \%$ and the Kappa coefficients is 0.91 . The fused image with Sentinel-1A and Landsat8 OLI is not only have more spatial information and spectral texture characteristics, but also enhances the distinguishing features of the images. The proposed method is beneficial to improve the accuracy and stability of remote sensing image classification.
\end{abstract}

\section{INTRODUCTION}

Remote sensing image is different from ordinary image, it not only contains R, G, B three visible band, but also extends to near-infrared, mid-infrared, far-infrared, microwave, etc.(Pohl and Genderen, 1998; Chen et al., 2006; Dey et al., 2010). Remote sensing image acquired by satellites, radars, unmanned aerial vehicles and other tools (Zhao and Zhong, 2013; Nencini et al., 2007; Zhou et al., 2017) are used to record and reflect the strong and weak information of the electromagnetic radiation of surface features(Du et al., 2012) according to a certain scale. Remote sensing image can not only objectively show the shape, size and color of the Earth's surface features, but also play an important role in the fields of land cover, farmland monitoring, natural disaster monitoring and emergency disaster prediction. At present, with the increasing resolution of remote sensing image, different requirements for the application and processing of remote sensing images are proposed in different occasions (Guo et al., 2016). The classification of remote sensing images, an important direction of remote sensing image processing and application, has been widely studied.

The early remote sensing image classification method mainly uses the visual interpretation of the researcher, depending on individual subjective experience, there are some disadvantages such as classification error, susceptible to many factors and etc. In order to overcome these weaknesses, the pattern recognition is introduced into the remote sensing image classification. The classification of remote sensing image classification based on pattern recognition is divided into supervised classification and unsupervised classification. The supervised classification includes the Minimum distance (Zhao, 2013), Mahalanobis distance method (Maesschalck et al., 2000), Parallelepiped classification, the neural network classification, the Support Vector Machine, the Fuzzy classification, Maximum likelihood method, etc. Unsupervised classification including K-means, ISODATA algorithm, etc. However, in the process of these classification algorithms, it still exists the phenomenon of misclassification and missing points, which leads to the final classification accuracy is not high. With the progress of image processing, artificial intelligence and deep learning (Murai and Omatu, 1997; Jin et al., 2006; Nencini et al., 2006; Zhao et al., 2010; Sheng, 2010; Peijun et al., 2011) have made new progress in the research of remote sensing image classification, but the remote sensing image classification is still have important research significance.

According to the application features of the three fusion level algorithm of remote sensing image, the feature level ( $\mathrm{Yu}$ and Qian, 1999; Yu et al., 2000; Ji et al., 2002; Qu et al., 2002; Li and Ma, 2007; Yan et al., 2008; Xiong et al., 2016; Ma et al., 2016; Zhai et al., 2017) is selected for experiment. Different types of remote sensing data, including MODIS images, SAR images, Landsat images and other hyperspectral remote sensing images, we selected Sentinel-1A and Landsat8 OLI images as data sources. By analyzing the overall classification precision and Kappa coefficients of the classified results(Gao et al., 2015; Zhang and Shen, 2016), the experimental results show that the fused image with Sentinel-1A and Landsat8 OLI not only have more spatial information and spectral texture characteristics, but also enhances the distinguishing features of the images. The proposed method is beneficial to improve the accuracy and stability of remote sensing image classification.

\footnotetext{
* Corresponding author
} 


\section{METHODOLOGY}

\subsection{The Overall Framework}

Firstly, Sentinel-1A and Landsat8 OLI should be preprocessed separately. And then selected the best fused image in three kind of fusion methods based on feature level (i.e., Gram-Schmidt spectral sharpening, Principal Component Analysis transform and Brovey transform) for the classification experimental. Finally, four kinds of image classification methods (i.e., Minimum distance, Mahalanobis distance method, Support Vector Machine and ISODATA classification) were selected in the classification process. The overall classification precision and the Kappa coefficient are used as the classification accuracy evaluation standard, compare the fused image classification result.

\subsection{Remote Sensing Images Preprocessing}

In the process of remote sensing image, sensor errors, the difference of atmosphere scattering and illumination condition may cause radiation error, geometrical distortion and other phenomena. Before the fusion of remote sensing images, Sentinel-1A and Landsat8 OLI images should be preprocessed separately.

The preprocessing of Sentinel-1A image is as follows: (1)The Sentinel 1A image data is imported into the SARscape software to generate the SARscape recognized data format.

(2)Because SAR is a coherent system, we need to suppress speckle noise, and do long sight and filter processing.

(3)In order to do image fusion based on feature level, we get the backscatter coefficients of images through geocoding and radiometric calibration, and the image is projected into Gausskruger coordinate system with DEM.

The preprocessing of Landsat8 OLI image is as follows: (1)Locate the file named LC81230322017319LGN00 MTL.txt, import Landsat8 OLI image in Envi 5.3, and use linear2\% to enhance the image of multispectral image to enhance visual effect.

(2)A radiometric calibration of the image is performed to convert the $\mathrm{DN}$ value to the actual radiant brightness value.

(3)The three-time convolution interpolation method is used for geometric correction, which eliminates the error caused by the non-system factors (such as the sensor's posture and high instability).

\subsection{Remote Sensing Image Fusion}

Remote sensing image fusion is the technology and framework system of higher quality data, more optimized features and more reliable knowledge through multi-level organic combination matching, analysis and decision making of various remote sensing images (Yuan and Wang, 2005; Du et al., 2016). Remote sensing image fusion processing mainly includes three levels (i.e., pixel level, feature level and decision level). The pixel level is a direct algebraic operation between pixels, and then extracts the feature information of the object after processing and analyzing. The advantage is that it can preserve the fine information of the image, but the processing information is large and time-consuming. The image fusion based on feature level is to deal with the original data, such as sensors with different spatial and temporal resolutions (i.e., MODIS, SAR, Landsat TM and so on) to extract the feature information and to integrate the same type of image. Image fusion can generate new images with higher confidence, and realize information compression. Image fusion based on decision level is to identify, classify or target the image information first, and then to integrate the acquired thematic image after obtaining the decision information such as the regional feature and the target state (Zhou et al., 2003). According to the application features of the three level algorithm of remote sensing image fusion, the feature level is selected to carry out the experiment.

In the remote sensing image fusion algorithm based on feature level, such as Brovey transform, HSV transform, Principal Component Analysis transform, Color Normalization transform, Gram-Schmidt spectral sharpening, etc. We select three feature level fusion methods (i.e., Gram-Schmidt spectral sharpening, Principal Component Analysis transform and Brovey transform) to do the contrast experiment, then select the best fusion image for classification experiments.

\subsection{Remote Sensing Images Classification}

The objective of remote sensing images classification is to classify each pixel in the image according to its spectral luminance value at different bands, spatial structure characteristics (i.e., image texture, shape, directionality, complexity) or other ancillary information, according to a rule or algorithm divided into different categories, so that each pixel attach a unique identity. The most commonly used classification methods of remote sensing images are supervised classification and unsupervised classification.

However, in the process of remote sensing images classification, there still exists the phenomenon of misclassification and missing points, which leads to the final classification accuracy is not high. On the one hand, because of the high dimension characteristic, uncertainty, information redundancy and the same spectral heterogeneity of the ground cover and the common foreign body (Gao et al., 2015), the remote sensing image data structure is highly nonlinear, and some classification methods based on statistical pattern recognition are difficult to be identified directly. On the other hand, in the field of remote sensing image supervision classification, the number of prior samples is limited and the quality is not uniform, the parameters of classifier model cannot be estimated or inaccurate. These problems lead to more and more complex feature extraction algorithms, more and more mathematical models, more and more advanced classification models, higher classification accuracy, but only in the level of data processing upgrade and progress, in information extraction, knowledge delivery has yet to be improved. With the progress of image processing, the current pattern recognition, neural network, computer vision and others have become the important technical means of hyperspectral remote sensing image classification. Some new methods, such as expert system and deep learning, have made a breakthrough in the research of remote sensing image classification. Based on the feature level fusion, we choose four kinds of image classification methods (i.e. Minimum distance, Mahalanobis distance method, Support Vector Machine and ISODATA classification) to do contrast experiment.

\section{EXPERIMENTAL RESULTS AND ANALYSIS}

\subsection{Study Area and Data}

In this paper, we selected Sentinel-1A and Landsat8 OLI images as data sources, and use ENVI 5.3, Google Earth and ArcGIS 
software to process remote sensing images. We choose Beijing, China as the research area, the located in northern latitude $39^{\circ} 54^{\prime} 20^{\prime \prime}$, east longitude $116^{\circ} 25^{\prime} 29^{\prime \prime}$. North of the North China Plain, backed by Yanshan Mountain, the climate for the typical northern temperate sub-humid continental monsoon climate. The Sentinel-1A images imaging time is January 27, 2018, the center longitude is $39^{\circ} 45^{\prime} 12^{\prime \prime}$, the center latitude is $115^{\circ} 44^{\prime} 14^{\prime \prime}$, the polarization mode is $\mathrm{VH}$; Landsat8 OLI images imaging time is November 15, 2017, The center longitude is $116^{\circ} 6^{\prime} 10^{\prime \prime}$ and the center latitude is $40^{\circ} 19^{\prime} 58^{\prime \prime}$. The experimental areas include water, farmland, greenbelt, barren, road traffic and other typical types of features. $1,2,3,4,5,6,7,9$ are eight multispectral bands with a resolution of $30 \mathrm{~m}$, OLI8 for panchromatic band image with a resolution of $15 \mathrm{~m}$. Other data include the Beijing land Use status map (2006-2020), the Google Earth high score image and etc. The geographical location of study area is shown in Figure 1.

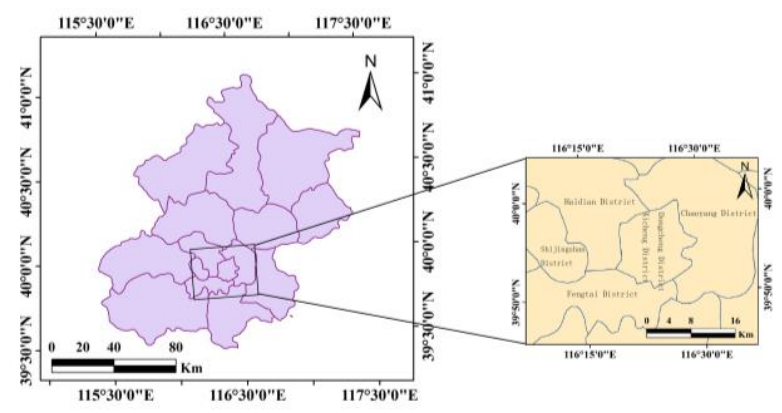

Figure 1. Geographical location of the study area

\subsection{The Results of Image Fusion}

After preprocessing Sentinel-1A and Landsat8 OLI images, Gram-Schmidt spectral sharpening, Principal Component Analysis transform and Brovey transform were performed. Refer to the Google Earth topographic map, we can found that: Gram-Schmidt spectral sharpening effect is the best, can retain geomorphic texture characteristics, image on the demarcation line between different features are very clear. From the view of color, the same kind of object color is basically consistent.

The Brovey transform effect is inferior to the Gram-Schmidt spectral sharpening, the overall picture color becomes shallow. The Principal Component Spectral sharpening and GramSchmidt spectral sharpening effect is similar, the spectral characteristics are obvious, but the Principal Component Analysis transform is time-consuming, to the terrain detail performance still need to improve. In conclusion, the GramSchmidt spectral sharpening is superior to the other two fusion methods in maintaining spectral properties. The results of the three fusion methods are shown in Figure 2.
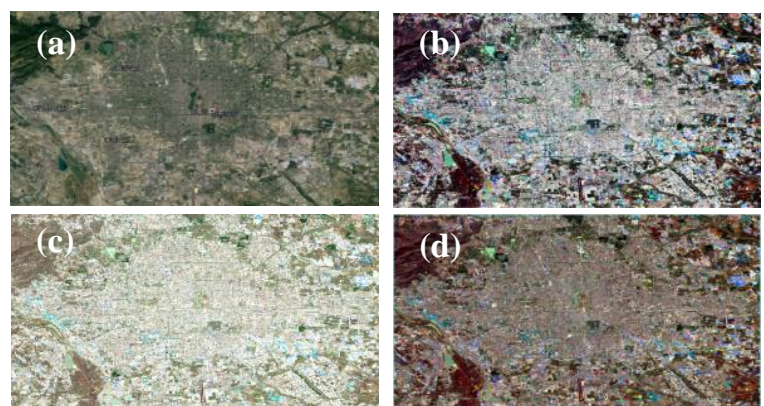

Figure 2. The results of three fusion methods. (a) Google Earth topographic map; (b) Gram-Schmidt spectral sharpening; (c) Principal Component Analysis transform; (d) Brovey transform.

\subsection{The Results of Image Classification and Analyzed}

Because the classification accuracy is affected by the quality of the training samples, the separation between the samples is more than 1.9 (Tian et al., 2008, Lou et al., 2016), which indicates that the training samples are qualified for the experiment and can be used to classify the fused images. The segregation of training samples is shown in Table 3.

\begin{tabular}{ccccccc}
\hline & Waters & Farmland & Greenbelt & Barren & $\begin{array}{l}\text { Road } \\
\text { traffic }\end{array}$ & $\begin{array}{c}\text { Constru- } \\
\text { ction land }\end{array}$ \\
\hline Waters & 1 & 1.9998 & 1.9583 & 1.9283 & 1.9445 & 1.9831 \\
Farmland & 1.9998 & 1 & 1.9387 & 1.9484 & 2 & 1.9293 \\
Greenbelt & 1.9583 & 1.9387 & 1 & 1.9780 & 1.9108 & 1.9812 \\
Barren & 1.9283 & 1.9484 & 1.9780 & 1 & 1.9779 & 1.9953 \\
$\begin{array}{c}\text { Road } \\
\text { traffic }\end{array}$ & 1.9445 & 2 & 1.9108 & 1.9779 & 1 & 1.9479 \\
$\begin{array}{c}\text { Construct- } \\
\text { ion land }\end{array}$ & 1.9831 & 1.9293 & 1.9812 & 1.9953 & 1.9479 & 1 \\
\hline & & & & & & \\
\hline
\end{tabular}

Table 3. The segregation of training samples

In this paper, three kinds of supervised classification algorithms (i.e. Minimum distance, Mahalanobis distance method, Support Vector Machine) and one unsupervised classification algorithm, such as ISODATA classification is selected to do experimental. The results of the four classification methods are shown in Figure 4.
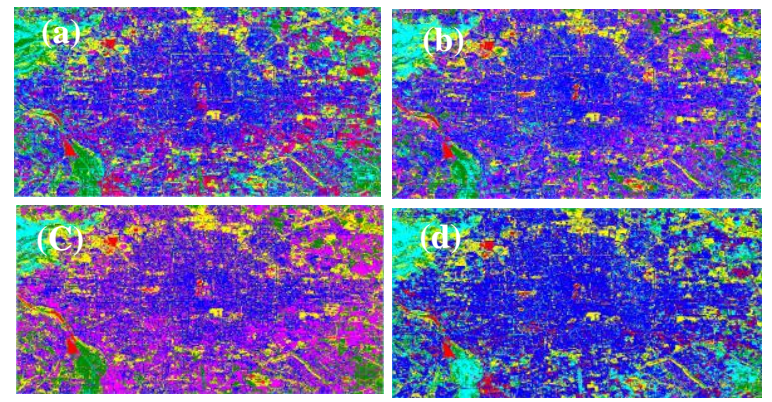

Figure 4. The results of the four classification methods.

(a) Minimum distance; (b) Mahalanobis distance method; (c) Support Vector Machines; (d) ISODATA classification.

According to the standard land use chart of Beijing, we can find that each method has advantages, four kinds of classification methods have different interpretations to the topography distribution of Beijing Central District. In order to facilitate the qualitative evaluation of the classification results, six typical features were selected (i.e. waters, farmland, greenbelt, barren, road traffic, construction land).

Because the principle of the four classification methods is different, the effect on the classification of features is also more distinct. In the classification of each features, the overall classification precision is different, such as the Minimum distance method for road traffic classification effect is better, Mahalanobis distance method to the waters classification effect is good, the Support Vector Machine to the waters, the greenbelt, the road traffic classification effect is relatively high, the ISODATA classification to the construction land classification effect is best. In conclusion, the Support Vector Machine classification result compared to the other three 
methods, more close to the standard land use, is the best algorithm in four kinds of classification method. The overall classification precision and Kappa coefficients of the four kinds of method are shown in Figure 5.

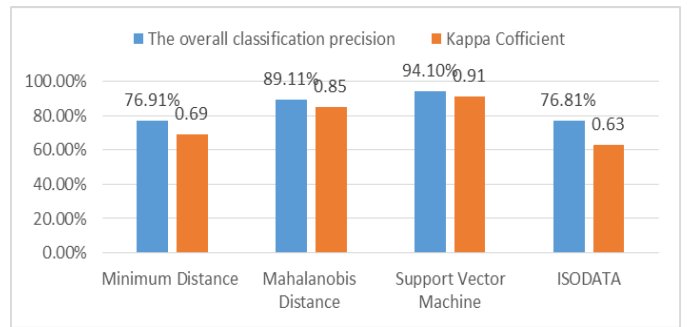

Figure 5. The overall classification precision and Kappa coefficients of the four kinds of method

\section{CONCLUSION}

In this paper, we selected the latest data images of Sentinel-1A and Landsat8 OLI images as data sources, choose Beijing, China as the study area, and propose a classification method of remote sensing images based on feature level fusion. Compare and analyze three kind of feature level fusion methods (i.e., Gram-Schmidt spectral sharpening, Principal Component Analysis transform and Brovey transform), and then selected the best fused image for the classification experiment. In the classification process, we choose four kinds of image classification methods (i.e. Minimum distance, Mahalanobis distance method, Support Vector Machine and ISODATA classification ) to do the contrast experiment. According to the standard land use classification image, and classification accuracy evaluation criteria such as the overall classification precision and Kappa coefficient, it is analyzed in the remote sensing image processing and application whether the image fusion processing is advantageous before the classification.

The experimental results show that the fusion image of Sentinel-1A image and Landsat8 OLI image is better by GramSchmidt spectral sharpening, the overall classification precision of the fused image in four classification algorithms is above $76 \%$, and the Kappa coefficients is above 0.6.In four kinds of classification algorithms, the fusion image has the best applicability to SVM classification, the overall classification precision is $94.01 \%$ and Kappa coefficients is 0.91 .

In the future, we will further study how to make full use of spectral information, geometric structure information and spatial information to classify the fused images based on the existing classification process to improve accuracy. Gradually jump out of a single data processing and image classification, and study the integration of various classifiers, such as supervised and unsupervised integration.

\section{REFERENCES}

Pohl C, Genderen J L V., 1998. Review article Multisensor image fusion in remote sensing: Concepts, methods and applications. International Journal of Remote Sensing, 19(5), 823-854.

Chen X L, Zhao H M, Li P X, et al., 2006. Remote sensing image-based analysis of the relationship between urban heat island and land use/cover changes. Remote Sensing of Environment, 104(2), 133-146.
Dey V, Zhang Y, Zhong M, 2010. A review on image segmentation techniques with remote sensing perspective. Pattern Recognition, 38(9), 1277-1294.

Zhao B, Zhong Y, 2013. High Spatial Resolution Remote Sensing Image Segmentation Based on Multi-agent Theory. Acta Geodaetica Et Cartographica Sinica, 113(1).

Nencini F, Garzelli A, Baronti S, et al., 2007. Remote sensing image fusion using the curvelet transform. Information Fusion, 8(2), 143-156.

Zhou L, Guo J, Hu J, et al., 2017. Wuhan Surface Subsidence Analysis in 2015-2016 Based on Sentinel-1A Data by SBASInSAR. Remote Sensing, 9(10), 982.

Du P, Xia J, Zhang W, et al., 2012. Multiple classifier system for remote sensing image classification: a review. Sensors, 12(4), 4764-4792.

Guo J, Zhou L, Yao C, et al., 2016. Surface Subsidence Analysis by Multi-Temporal InSAR and GRACE: A Case Study in Beijing. Sensors, 16(9).

Zhao Y S, 2013. Remote Sensing Application Analysis Principles and Method, Science Press.

Maesschalck R D, Jouan-Rimbaud D, Massart D L., 2000. The Mahalanobis distance. Chemometrics \& Intelligent Laboratory Systems, 50(1), 1-18.

Murai H, Omatu S., 1997. Remote sensing image analysis using a neural network and knowledge-based processing. International Journal of Remote Sensing, 18(4), 811-828.

$\mathrm{Su} \mathrm{H}$ J, Sheng Y H, 2010. Hyperspectral feature recognition based on kernel PCA and relational perspective map. Chinese Optics Letters, 8(8), 811-814.

Du P J, Zhang W, Zhang S B, et al., 2011. Hyperspectral remote sensing image classification based on decision level fusion. Chinese Optics Letters, 9(3), 33-36.

Zhao Q, Song W, Sun G, 2010. The Recognition of Land Cover with Remote Sensing Image Based on Improved BP Neutral Network. In: International Conference on Multimedia Technology. IEEE, 1-4.

Jin hui L I, Cheng hui L I, Bo W U, 2006. Classification of Remote Sensing Images Based on Sample Purification and BP Neutral Networks. Journal of Yangtze River Scientific Research Institute, 23(6), 51-54

Nencini F, Garzelli A, Baronti S, et al., 2006.Remote sensing image fusion using the curvelet transform. Information Fusion, 8(2), 143-156.

Zhai A, Wen X, Xu H, et al., 2017. Multi-Level Model Based on Multi-Scale and Multi-Feature Fusion for SAR Image. Remote Sensing, 9(11), 1085.

Li H, Ma G, 2007. Support Vector Machine Based Approach for Feature-Level Multiple Source and Heterogeneity Data Fusion. Journal of North China Institute of Aerospace Engineering.

Xiong J, Li F, Liu J, 2016. Fusion of Different Height Pyroelectric Infrared Sensors for Person Identification. IEEE Sensors Journal, 16(2), 436-446.

Ma G, Yang X, Zhang B, et al., 2016. Multi-feature fusion deep networks. Neurocomputing, 218(C), 164-171.

Yu X L, Qian G H, Jia X G, et al., 2000. Multi-spectral and SAR Remote Sensing Image Fusion Classification Feature Selection. Journal of Infrared and Millimeter Waves, 19(6), 449-453.

Yu X L, Qian G J, 1999. Comparison of Fusion and Classification of TM and SAR Remote Sensing Images at Different Levels. Remote Sensing Technology and Application, 14(3), 38-43.

Qu J S, Wang C, Wang Z Z, 2002. Data Fusion Based Technology for Remote Sensing Image Processing. Journal of Image and Graphics, 7(10), 985-993. 
Yan M, Wan T, Feng C, et al., 2008. Inference for Kappas for Longitudinal Study Data: Applications to Sexual Health Research. Biometrics, 64(3).

Gao W J, Wang J L, Liu G J, 2015. Effects of Training Samples on Remote Sensing Image Classification Accuracy. Yunnan Geographic Environment Research, 27(2), 31-36.

Zhang L P, Shen H F, 2016. Progress and Prospect of Remote Sensing Data Fusion. Journal of Remote Sensing, 20(5), 10501061.

Du P J, Xia J S, et al., 2016. Research Progress of Hyperspectral Remote Sensing Image Classification. Journal of Remote Sensing, 20(2), 236-256.

Yuan J G, Wang W, 2005. Application of Multi-source Remote Sensing Data Fusion. Journal of Geo-Information Science, 7(3), 97-103.

Zhou Q, Li F, Zhang H T, et al., 2003. Application of Supervised Classification Technology in High Resolution Satellite Imagery. Journal of Beijing Forestry University, (s1), 47-49.

Gao W J, Wang J L, Liu G J, 2015. Effects of Training Samples on Remote Sensing Image Classification Accuracy. Yunnan Geographic Environment Research, 2015, 27(2), 31-36.

Tian Y, Tassi L T, Ding J L, et al., 2008. Land Cover Classification Based on Support Vector Machines. Resources Science, 30(8), 1268-1274.

Zhai T L, Jin G, Deng X Z, et al., 2016. Land use classification method based on multi-source remote sensing image fusion in Wuhan City. Resources and Environment in the Yangtze Basin, 25(10), 1594-1602. 